\title{
GLOBAL BOUNDARY CONTROLLABILITY OF THE DE ST. VENANT EQUATIONS BETWEEN STEADY STATES
}

\author{
M. GUGAT ${ }^{1}$, G. LEUGERING * \\ Technische Universität Darmstadt Fachbereich Mathematik, AG10, Schlossgartenstr. 7, \\ 64289 Darmstadt, Germany
}

Received 9 March 2000, revised 8 August 2001

ABSTRACT. - We consider the problem of exactly controlling the states of the de St. Venant equations from a given constant state to another constant state by applying nonlinear boundary controls. During this transition the solution stays in the class of $C^{1}$-solutions. There are no restrictions on the distance between the initial state and the target state, so our result is a global controllability result for a nonlinear hyberbolic system.

(C) 2003 L'Association Publications de l'Institut Henri Poincaré. Published by Elsevier B.V. All rights reserved

MSC: 93C20; 93B05; 76B75

Keywords: Global controllability; Nonlinear hyperbolic system; de St. Venant equation; Characteristic form

RÉSUMÉ. - On s'intéresse ici à un système décrit par les equations de St. Venant. On montre qu'on peut contrôler le système d'un état initial constant à un autre état constant avec des contrôles frontières non linéaires de façon qu'il y ait une solution $C^{1}$.

Il n'y a pas de restriction concernant la distance entre les données initiales et les données finales, donc notre résultat est un résultat de contrôlabilité globale exacte pour un système hyperbolique non linéaire.

(C) 2003 L'Association Publications de l'Institut Henri Poincaré. Published by Elsevier B.V. All rights reserved

Mots Clés: Contrôlabilité exacte globale; Équations de St. Venant

\section{Introduction}

We consider the flow through a frictionless horizontal channel that is described by the de St. Venant equations (see [14]). Cirina (see [2]) has shown local null controllability

\footnotetext{
* Corresponding author.

E-mail addresses: gugat@mathematik.tu-darmstadt.de (M. Gugat), leugering@mathematik.tu-darmstadt.de (G. Leugering).

${ }^{1}$ Supported by DFG-research cluster: real-time optimization of complex systems; grant number Le595/13-1.
} 
for nonlinear hyperbolic systems. In a more recent paper [12], Li, Rao and Jin established local controllability for a quasilinear strictly hyperbolic system (see also [13]).

Stabilizability of open channel flow around an equilibrium, described by the de St. Venant equations, has been shown by Coron, d'Andrea-Novel and Bastin in [4]. Finally, in [10], Leugering and Schmidt have shown local exact controllability and local stabilizability for networks of canals described by the de St. Venant equations (see also [7]).

In this paper, we present a result on global controllability of the de St. Venant equations with no restrictions on the distance between the initial state and the target. As stated for example in Dafermos [6], p. 140, classical solutions generally break down in finite time as a result of collision of characteristics (see also [11]). However, we show that starting from a constant subcritical initial state we can reach any other constant subcritical state in finite time with boundary controls that can be chosen in such a way that shocks are avoided and the state remains within the context of classical solutions.

In order to avoid shocks, it is essential to change the state sufficiently slow which is possible if the control period is chosen sufficiently long.

Apart from its mathematical content, our result appears to be relevant for applications where shocks have to be avoided, which is particularily true for drainage- and sewer systems. Our proofs are based on the characteristic form of the equations. They are constructive and show how controls can actually be computed numerically. As the boundary controls are not unique, there is some flexibility with respect to optimization.

Our result is global in the sense that it holds for initial states and terminal states that can be arbitrarily far away from each other. This is in contrast to the local results given in the literature that hold for initial states in a neighbourhood of the desired terminal state only.

Other problems of global controllability have been studied by E.J.P.G. Schmidt in [15, 16]

\section{Definitions and notation}

We consider the problem of boundary control of the flow through a frictionless horizontal channel, where the boundary conditions are given in terms of the Riemann invariants. The channel is parametrized lengthwise by $x \in[0, L]$. Let $U(t, x)$ and $a(t, x)$ denote the average velocity over the cross section of the channel and the wetted area at $x$ at time $t$, respectively. Furthermore, let $b(x, a)$ be the width of the water surface and $g$ be the gravitational constant. Then the de St. Venant equations governing the flow of water in that channel are given in form of the following quasilinear hyperbolic system:

$$
\partial_{t}\left(\begin{array}{c}
a \\
U
\end{array}\right)+\left(\begin{array}{cc}
U & a \\
g / b(a) & U
\end{array}\right) \partial_{x}\left(\begin{array}{c}
a \\
U
\end{array}\right)=0 .
$$

A detailed derivation of the model can be found in [5,8]. Let $c(t, x)=[g a(t, x) /$ $b(x, a(t, x))]^{1 / 2}$ denote the corresponding wave celerity. Define the Riemann invariants 
$R^{+}=U+2 c, R^{-}=U-2 c$ and

$$
A\left(R^{+}, R^{-}\right):=\left(\begin{array}{cc}
\frac{3}{4} R^{+}+\frac{1}{4} R^{-} & 0 \\
0 & \frac{1}{4} R^{+}+\frac{3}{4} R^{-}
\end{array}\right)=\left(\begin{array}{cc}
U+c & 0 \\
0 & U-c
\end{array}\right) .
$$

The de St. Venant equations can be written in the form

$$
\partial_{t}\left(\begin{array}{l}
R^{+} \\
R^{-}
\end{array}\right)+A\left(R^{+}, R^{-}\right) \partial_{x}\left(\begin{array}{l}
R^{+} \\
R^{-}
\end{array}\right)=0 .
$$

These equations form a nonlinear hyperbolic system of the form considered in Cirina [2,3] with $z=\left(R^{+}, R^{-}\right)$and the diagonal matrix $A$ and $f=0$.

For a matrix $M \in R^{2 \times 2}$, define $|M|=\max \left\{\left|m_{11}\right|+\left|m_{12}\right|,\left|m_{21}\right|+\left|m_{22}\right|\right\}$. For $r \in R^{2}$ let $|r|=\max \left\{\left|r_{1}\right|,\left|r_{2}\right|\right\}$.

We have $|A|=\max \left\{\left|\frac{3}{4} R^{+}+\frac{1}{4} R^{-}\right|,\left|\frac{1}{4} R^{+}+\frac{3}{4} R^{-}\right|\right\}$. The norms of the first partial derivatives of $A$ are bounded above by 1 .

In Cirina's papers [2,3] the classes $\Sigma(\mathcal{R}, m, \alpha, \delta, k)$ are defined and play an important role.

Our system is in diagonal form, hence in the notation of Cirina we have $(A, f) \in$ $\Sigma(R \times[0, \infty), 2, \alpha, 1 / 2, k)$ if $k \geqslant 1$ is such that $|A| \leqslant k$ for all $\left(R^{+}, R^{-}\right)$with $\left|\left(R^{+}, R^{-}\right)\right| \leqslant \alpha$. Here $f=0$ and the matrix $S$ in the definition of the class can be chosen as the identity matrix. The condition $|A| \leqslant k$ is satisfied for example if $\frac{3}{4}\left(\left|R^{+}\right|+\right.$ $\left.\left|R^{-}\right|\right) \leqslant k$.

\section{A local result}

In this section we start with a result about local controllability.

The results given in the literature ususally state that we can steer the system from a given initial state to a target state, provided that they are sufficiently close to each other.

In this section we state a result of a different type. It guarantees that for each element of a compact set of initial states it is possible to reach target states in balls of the same size around the initial states within the same control time. So our result could be described as a result about uniform local controllability. For this result it is essential that a uniform upper bound for the eigenvalues of the system matrix is valid.

Later we will use this result to prove that global controllability is possible, by constructing a global control from a finite number of local controls. This is possible since the following theorem ensures that for a given small distance the time intervals needed to go from a point to a target point within that distance are uniformly bounded.

THEOREM 1. - Consider the de St. Venant system (1) with boundary conditions of the form $R^{+}(t, 0)=g_{1}(t)$ and $R^{-}(t, L)=g_{2}(t)$. Let a nonempty compact rectangular set $\Omega=\left[a_{+}, b_{+}\right] \times\left[a_{-}, b_{-}\right] \subset R^{2}$ be given such that for all $\left(R^{+}, R^{-}\right) \in \Omega$, we have $3 / 4 R^{+}+1 / 4 R^{-}>0$ and $1 / 4 R^{+}+3 / 4 R^{-}<0$, that is $\Omega$ contains only subcritical states.

Let $\delta>0$ be given. Define

$$
T=\max _{\left(R^{+}, R^{-}\right) \in \Omega} \max \left\{\frac{L+\delta}{\left|(3 / 4) R^{+}+(1 / 4) R^{-}\right|}, \frac{L+\delta}{\left|(1 / 4) R^{+}+(3 / 4) R^{-}\right|}\right\} .
$$


Then there exists a number $\alpha>0$, such that for all constant initial states $\left(R_{1}^{+}, R_{1}^{-}\right) \in \Omega$ and for all constant terminal states $\left(R_{2}^{+}, R_{2}^{-}\right) \in \Omega$ with

$$
\max \left\{\left|R_{1}^{+}-R_{2}^{+}\right|,\left|R_{1}^{-}-R_{2}^{-}\right|\right\} \leqslant \alpha,
$$

the boundary controls $g_{1}, g_{2}$ can be chosen such that in time $T$, the system has reached the terminal state $\left(R_{2}^{+}, R_{2}^{-}\right)$and the corresponding solution is continuously differentiable.

Moreover, by choosing $\alpha$ sufficiently small, the maximal absolute values of the derivatives

$$
\partial_{x} R^{+}(t, x), \quad \partial_{x} R^{-}(t, x), \quad \partial_{t} R^{+}(t, x), \quad \partial_{t} R^{-}(t, x), \quad g_{1}^{\prime}(t), \quad g_{2}^{\prime}(t)
$$

for $(t, x) \in[0, T] \times[0, L]$ can be made arbitrarily small.

Proof. - We define an initial state $\varphi: R \rightarrow R^{2}$ on the real line in the following way. For $x \in[0, L]$, let $\varphi(x)=\left(R_{1}^{+}, R_{1}^{-}\right)$. For $x \in(-\infty,-\delta] \cup[L+\delta, \infty)$ let $\varphi(x)=\left(R_{2}^{+}, R_{2}^{-}\right)$. For $x \in(-\delta, 0) \cup(L, L+\delta)$ define $\varphi$ in $C^{1}(R)$, such that the components $\varphi_{1}$ and $\varphi_{2}$ of $\varphi$ are monotone functions on $(-\delta, 0)$ and on $(L, L+\delta)$ and

$$
\max _{x \in R}\left\{\left|\varphi_{1}^{\prime}(x)\right|,\left|\varphi_{2}^{\prime}(x)\right|\right\} \leqslant \frac{3}{\delta} \max \left\{\left|R_{1}^{+}-R_{2}^{+}\right|,\left|R_{1}^{-}-R_{2}^{-}\right|\right\} .
$$

Since the set $\Omega$ is compact, we can choose a number $c_{0}>0$ such that for all $\left(R^{+}, R^{-}\right) \in$ $\Omega$ we have $\left|\left(R^{+}, R^{-}\right)\right| \leqslant c_{0}$. Then we can choose a number $k \geqslant 1$ such that for all $\left(R^{+}, R^{-}\right)$with $\left|\left(R^{+}, R^{-}\right)\right| \leqslant 2 c_{0}$ we have $\left|A\left(R^{+}, R^{-}\right)\right| \leqslant k$. Define $a=-k T$ and $b=L+k T$. Then $(A, 0) \in \Sigma\left([a, b] \times[0, T], 2,2 c_{0}, 1 / 2, k\right)$. Thus Theorem 3.IV from Cirina [3], implies the existence of a number $\beta>0$ such that if

$$
\max _{x \in \mathbb{R}}\left\{\left|\varphi_{1}^{\prime}(x)\right|,\left|\varphi_{2}^{\prime}(x)\right|\right\} \leqslant \beta,
$$

the de St. Venant system with initial state $\varphi$ on $[a, b]$ has a unique continuously differentiable solution on the convex hull of the two sets $[a, b] \times\{0\}$ and $[0, L] \times[0, T]$. Let $\tau$ denote this region. The proof of Theorem 3.IV is based upon results by Hartman and Wintner whose proof can be found in [9].

Choose $\alpha \leqslant \beta \delta / 3$. Then (3) and (4) imply (5). Therefore by Theorem 3.IV from Cirina [3], a $C^{1}$ solution with initial state $\varphi$ on $[a, b]$ exists on the set $\tau$.

Moreover, Theorem 3.IV guarantees the existence of a constant $N>0$ such that for all $(x, t) \in[0, L] \times[0, T]$ we have

$$
\max \left\{\left|\partial_{x} R^{+}(t, x)\right|,\left|\partial_{x} R^{-}(t, x)\right|\right\} \leqslant N \max _{x \in \mathbb{R}}\left\{\left|\varphi_{1}^{\prime}(x)\right|,\left|\varphi_{2}^{\prime}(x)\right|\right\} \leqslant 3 N \alpha / \delta .
$$

(Note that in [3] there is a misprint in the statement of Theorem 3.IV, namely the inequality $\left|z_{x}\right| \leqslant N|\phi|$ instead of $\left|z_{x}\right| \leqslant N\left|\phi^{\prime}\right|$.)

This implies that by choosing $\alpha$ sufficiently small, we can make the absolute values of the derivatives $\partial_{x} R^{+}(t, x), \partial_{x} R^{-}(t, x)$ arbitrarily small. 
Since the initial state $\varphi_{1}$ has only values between $R_{1}^{+}$and $R_{2}^{+}$, this is also the case for the first component $R^{+}$of the solution on $[0, T]$. Similarly, $R^{-}$can only attain values between $R_{1}^{-}$and $R_{2}^{-}$.

Define

$$
M=\max _{(x, t) \in[0, L] \times[0, T]}\left\{\left|(3 / 4) R^{+}+(1 / 4) R^{-}\right|,\left|(1 / 4) R^{+}+(3 / 4) R^{-}\right|\right\} .
$$

The system equation implies that

$$
\begin{aligned}
\max _{(x, t) \in[0, L] \times[0, T]}\left\{\left|\partial_{t} R^{+}\right|,\left|\partial_{t} R^{-}\right|\right\} & \leqslant M \max _{(x, t) \in[0, L] \times[0, T]}\left\{\left|\partial_{x} R^{+}\right|,\left|\partial_{x} R^{-}\right|\right\} \\
& \leqslant 3 M N \alpha / \delta .
\end{aligned}
$$

So we can also make the absolute values of the time-derivatives of $\left(R^{+}, R^{-}\right)$arbitrarily small by choosing $\alpha$ sufficiently small.

The slopes of the characteristic curves are given by $d x_{+} / d t=(3 / 4) R^{+}+(1 / 4) R^{-}$, and $d x_{-} / d t=(1 / 4) R^{+}+(3 / 4) R^{-}$, respectively. In the area of points above both of these characteristics, the solution $R^{+}$has the value $R_{2}^{+}$and $R^{-}$has the value $R_{2}^{-}$. By the definition of $T$ and on account of the rectangular shape of $\Omega$, this implies that at time $T$, the system has reached the terminal state $\left(R_{2}^{+}, R_{2}^{-}\right)$on the interval $[0, L]$.

This can be seen as follows. Since the set $\Omega$ contains only subcritical states, the $x_{+}$ characteristic curves have positive slope and the $x_{-}$curves have negative slope. Hence at time $T$, the characteristic with positive slope coming from the point $(t, x)=(0,-\delta)$ has reached the point $(T, L)$ and the characteristic with negative slope emanating from the point $(t, x)=(0, L+\delta)$ has reached the point $(T, 0)$.

We now define the functions $g_{1}, g_{2}$ by setting $g_{1}(t):=R_{+}(0, t)$ and $g_{2}(t):=R_{-}(L, t)$ and have thus constructed the required boundary controls.

We have $g_{1}^{\prime}(t)=\partial_{t} R_{+}(0, t)$ and $g_{2}^{\prime}(t)=\partial_{t} R_{-}(0, t)$. Hence the result that the absolute values of the derivatives of the control function can be made arbitrarily small by an appropriate choice of $\alpha$ also follows.

Remark 1.-To compute the functions $g_{1}$ and $g_{2}$ numerically, the initial value problem has to be solved with initial values given by the function $\varphi$ on the interval $[-\delta, L+\delta]$ with boundary conditions $R^{+}(t,-\delta)=R_{2}^{+}, R^{-}(t, L+\delta)=R_{2}^{-}$for $t \geqslant 0$. Of course, after time $T$ the computation can be stopped since for $t \geqslant T$ we have $g_{1}(t):=R_{+}(0, t)=R_{2}^{+}$, and $g_{2}(t)=R_{2}^{-}$.

Numerical Examples are given in Section 5.

Remark 2.-Theorem 1 is different from the local exact boundary controllability result that has been given in Theorem 3.1 in [12], since in Theorem 3.1, it is assumed that the $C^{1}$-norms of both the initial data and the final data are small, whereas in Theorem 1 it is only required that the norm of the difference between the initial and final data is sufficiently small.

\section{Global results}

We proceed to apply Theorem 1 to obtain a first result about global controllability. In this result, we consider rectangular sets that satisfy the assumptions of Theorem 1 . We 
show that we can steer the system from any point of such a set to any other point of this set in finite time.

THEOREM 2. - Let a nonempty compact rectangular set $\Omega=\left[a_{+}, b_{+}\right] \times\left[a_{-}, b_{-}\right] \subset$ $R^{2}$ be given such that for all $\left(R^{+}, R^{-}\right) \in \Omega$, we have

$$
3 / 4 R^{+}+1 / 4 R^{-}>0 \text { and } 1 / 4 R^{+}+3 / 4 R^{-}<0,
$$

that is $\Omega$ contains only subcritical states.

Then we can find boundary controls $g_{1}$ and $g_{2}$ that steer the de St. Venant system (1) with boundary conditions $R^{+}(t, 0)=g_{1}(t)$ and $R^{-}(t, L)=g_{2}(t)$ in finite time from any constant initial state in $\Omega$ to any other constant state in $\Omega$ in such a way that the corresponding solution is continuously differentiable.

Moreover, this can be achieved in such a way that the absolute values of the derivatives of the state $\left(R^{+}, R^{-}\right)$and of the controls $\left(g_{1}, g_{2}\right)$ remain smaller than any given upper bound.

Proof. - Let an initial point $A \in \Omega$ and a target point $B \in \Omega$ be given. For a natural number $n$ and $k \in\{0, \ldots, n\}$ define $\lambda_{k, n}=k / n$ and

$$
\omega_{k, n}=\left(1-\lambda_{k, n}\right) A+\lambda_{k, n} B .
$$

Since the set $\Omega$ is convex, the points $\omega_{k, n}$ are all elements of $\Omega$. Now choose $n$ sufficiently large such that for all $k \in\{1, \ldots, n\}$ for $\omega_{k, n}-\omega_{k-1, n}=(B-A) / n$ we have

$$
|B-A| / n \leqslant \alpha,
$$

where $\alpha$ is as in Theorem 1 . Now Theorem 1 implies that for all $k \in\{1, \ldots, n\}$, it is possible to steer the system from $\omega_{n, k-1}$ to $\omega_{n, k}$ in time $T$ as defined in (2).

So we can steer the system from $A=\omega_{0, n}$ to $\omega_{1, n}$, from $\omega_{1, n}$ to $\omega_{2, n}, \ldots$ and finally from $\omega_{n-1, n}$ to $\omega_{n, n}=B$ and after time $n T$ the system has reached the desired terminal state $B$.

Moreover, Theorem 1 yields controls for which the absolute values of the derivatives of the state $\left(R^{+}, R^{-}\right)$and of the controls $\left(g_{1}, g_{2}\right)$ can be made smaller than any given upper bound by choosing $\alpha$ sufficiently small.

Of course, if $\alpha$ is small, the number $n$ has to be chosen large, so for a small bound for the norm of the derivatives, the time needed to steer the system to the target state is long.

We are now ready to prove that it is possible to steer the system from any constant subcritical state to any other constant subcritical state in finite time while avoiding the formation of shocks.

THEOREM 3. - From a constant subcritical state $\left(R^{+}, R^{-}\right)$(i.e. $3 / 4 R^{+}+1 / 4 R^{-}>0$ and $\left.1 / 4 R^{+}+3 / 4 R^{-}<0\right)$ the system can be steered to any other constant subcritical state by boundary controls in finite time with a continuously differentiable state.

Moreover, for every given positive upper bound the system can be steered in such a way that the maximum norms of the derivatives of the state and of the controls remain below this bound. 
Proof. - Let two constant subcritical states $\left(R_{A}^{+}, R_{A}^{-}\right)$and $\left(R_{B}^{+}, R_{B}^{-}\right)$be given.

As in the proof of Theorem 2 , for $n \in \mathbb{N}$ and $k \in\{0, \ldots, n\}$ define $\lambda_{k, n}=k / n$ and let

$$
R_{k, n}^{+}=\left(1-\lambda_{k, n}\right) R_{A}^{+}+\lambda_{k, n} R_{B}^{+}, \quad R_{k, n}^{-}=\left(1-\lambda_{k, n}\right) R_{A}^{-}+\lambda_{k, n} R_{B}^{-} .
$$

Then $R_{0, n}^{+}=R_{A}^{+}, R_{0, n}^{-}=R_{A}^{-}, R_{n, n}^{+}=R_{B}^{+}, R_{n, n}^{-}=R_{B}^{-}$.

If the number $n$ is chosen sufficiently large, for all $k \in\{1, \ldots, n\}$ the nonempty sets

$$
\Omega_{k}=\left[\min \left\{R_{k-1}^{+}, R_{k}^{+}\right\}, \max \left\{R_{k-1}^{+}, R_{k}^{+}\right\}\right] \times\left[\min \left\{R_{k-1}^{-}, R_{k}^{-}\right\}, \max \left\{R_{k-1}^{-}, R_{k}^{-}\right\}\right]
$$

contain only subcritical states, hence for all $k \in\{1, \ldots, n\}$ Theorem 2 is applicable to $\Omega_{k}$. So we can steer the system from the initial state $\left(R_{A}^{+}, R_{A}^{-}\right) \in \Omega_{1}$ to $\left(R_{1, n}^{+}, R_{1, n}^{-}\right) \in \Omega_{1}$, from $\left(R_{1, n}^{+}, R_{1, n}^{-}\right) \in \Omega_{2}$ to $\left(R_{2, n}^{+}, R_{2, n}^{-}\right) \in \Omega_{2}$ and so forth and finally from $\left(R_{n-1, n}^{+}, R_{n-1, n}^{-}\right) \in \Omega_{n}$ to the desired terminal state $\left(R_{B}^{+}, R_{B}^{-}\right) \in \Omega_{n}$, that we reach after $n$ such steps.

The assertion about the maximum norms of the derivatives and the controls also follows from Theorem 2.

Remark 3. - The combination of Theorem 3 with a result about local controllability (see $[12,10]$ ) yields a result about controllability from states that are contained in a small ball around a constant subcritical state to states that are in a small ball around another constant subcritical state.

\section{Numerical examples}

Example 1. - Now we want to define explicitly a function $\varphi$ as used in the proof of Theorem 1. Let $\delta>0$ and $\beta \in \mathbb{R}$ be given. Define

$$
p(x)=\frac{6 \beta}{\delta^{3}}\left(\delta \frac{x^{2}}{2}-\frac{x^{3}}{3}\right) .
$$

Then $p(0)=0, p(\delta)=\beta, p^{\prime}(0)=0=p^{\prime}(\delta)$ and $p^{\prime}(x) \neq 0$ for all $x \in(0, \delta)$. Moreover, $\left|p^{\prime}(x)\right| \leqslant\left|p^{\prime}(\delta / 2)\right|=(3 / 2)|\beta| / \delta$ for all $x \in[0, \delta]$.

For $L>0$ define $L_{0}=L+\delta$. Define the function $\varphi$ with the components

$$
\varphi_{1}(x)= \begin{cases}R_{2}^{+}, & x \in(-\infty,-\delta), \\ R_{2}^{+}+p(x), & x \in[-\delta, 0) \text { with } \beta=R_{1}^{+}-R_{2}^{+}, \\ R_{1}^{+}, & x \in[0, L], \\ R_{2}^{+}+p\left(L_{0}-x\right), & x \in(L, L+\delta], \\ R_{2}^{+}, & x \in(L+\delta, \infty) .\end{cases}
$$

and

$$
\varphi_{2}(x)= \begin{cases}R_{2}^{-}, & x \in(-\infty,-\delta), \\ R_{2}^{-}+p(x), & x \in[-\delta, 0) \text { with } \beta=R_{1}^{-}-R_{2}^{-}, \\ R_{1}^{-}, & x \in[0, L], \\ R_{2}^{-}+p\left(L_{0}-x\right), & x \in(L, L+\delta], \\ R_{2}^{-}, & x \in(L+\delta, \infty) .\end{cases}
$$




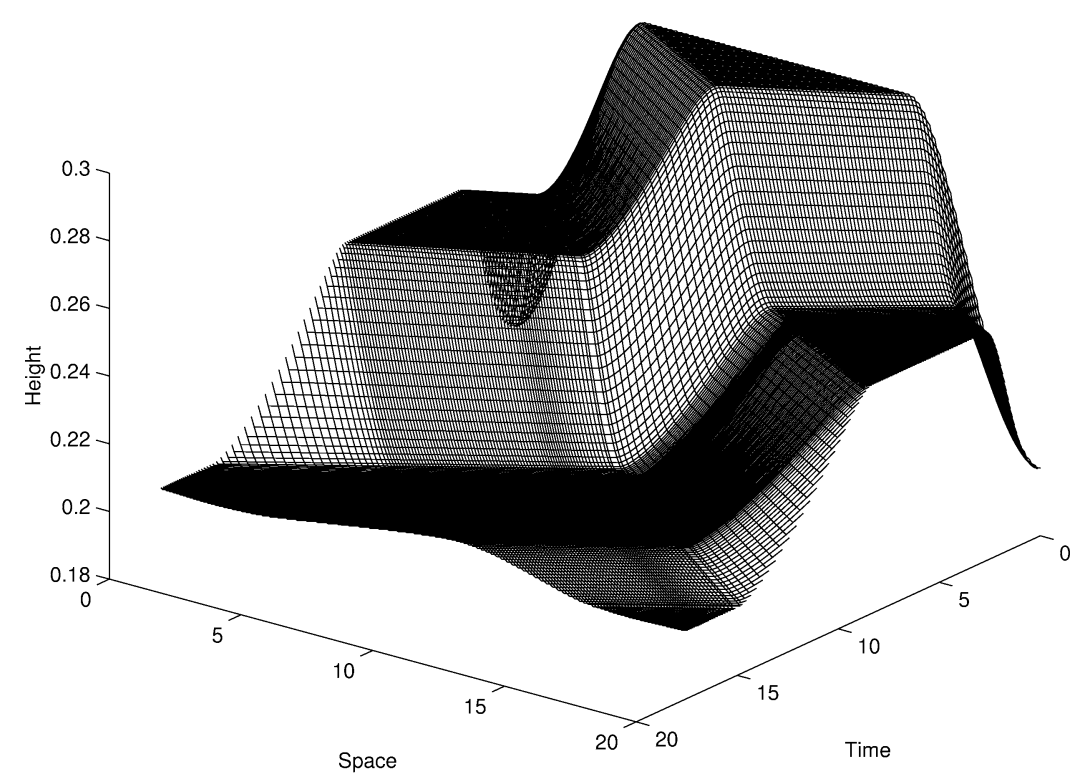

The water height $h$.

Fig. 1.

Then the function $\varphi=\left(\varphi_{1}, \varphi_{2}\right)$ satisfies the requirements of the proof of Theorem 1 .

For our numerical example, let $L=10, \delta=5$ and $R_{1}^{+}=2 \sqrt{0.3 g}$ with $g=9.81$, $R_{1}^{-}=-R_{1}^{+}, R_{2}^{+}=2 \sqrt{0.2 g}, R_{2}^{-}=-R_{2}^{+}$.

We consider a rectangular channel where for the water height $h$ we have $h=c^{2} / g$. So we start with water height 0.3 and zero velocity and want to steer the system to the state with water height 0.2 and zero velocity. Fig. 1 shows the water height $h=\left[0.25\left(R^{+}-R^{-}\right)\right]^{2} / g$ for $x$ in the interval $[-\delta, L+\delta]$ that is shifted to the interval $[0,20]$. So the interval $[0, L]$ is shown in the figure as the space interval $[5,15]$, the interval $[-\delta, 0]$ corresponds to $[0,5]$ and the interval $[L, L+\delta]$ to $[15,20]$.

Note that the initial time zero is in the back and the time 20 is in the front.

Example 2. - To illustrate the fact that for a fixed $\delta$ for the controls that we have constructed the derivatives of the state remain smaller if the distance between initial state and terminal state is smaller, we consider the data as in Example 1, but with terminal state $R_{2}^{+}=2 \sqrt{0.28 g}, R_{2}^{-}=-R_{2}^{+}$. So here we start with water height 0.3 and zero velocity and want to steer the system to the state with water height 0.28 and zero velocity.

Fig. 2 shows the height $h$ for a rectangular channel.

Example 3. - For an example where shock waves occur, we consider the data as in Example 2, but with $p=0$, hence

$$
\varphi_{1}(x)=\left\{\begin{array}{ll}
R_{2}^{+} & x \in[-\infty, 0), \\
R_{1}^{+} & x \in[0, L], \\
R_{2}^{+} & x \in(L, \infty)
\end{array} \quad \text { and } \quad \varphi_{2}(x)= \begin{cases}R_{2}^{-} & x \in[-\infty, 0), \\
R_{1}^{-} & x \in[0, L], \\
R_{2}^{-} & x \in(L, \infty)\end{cases}\right.
$$




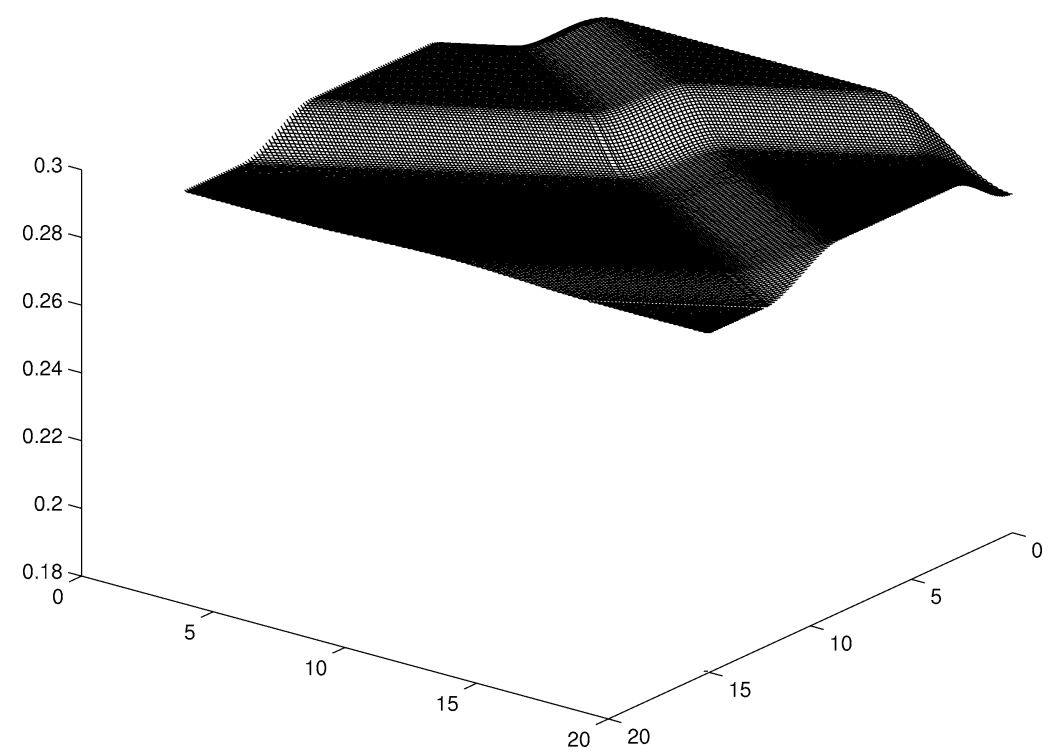

The water height $h$.

Fig. 2.

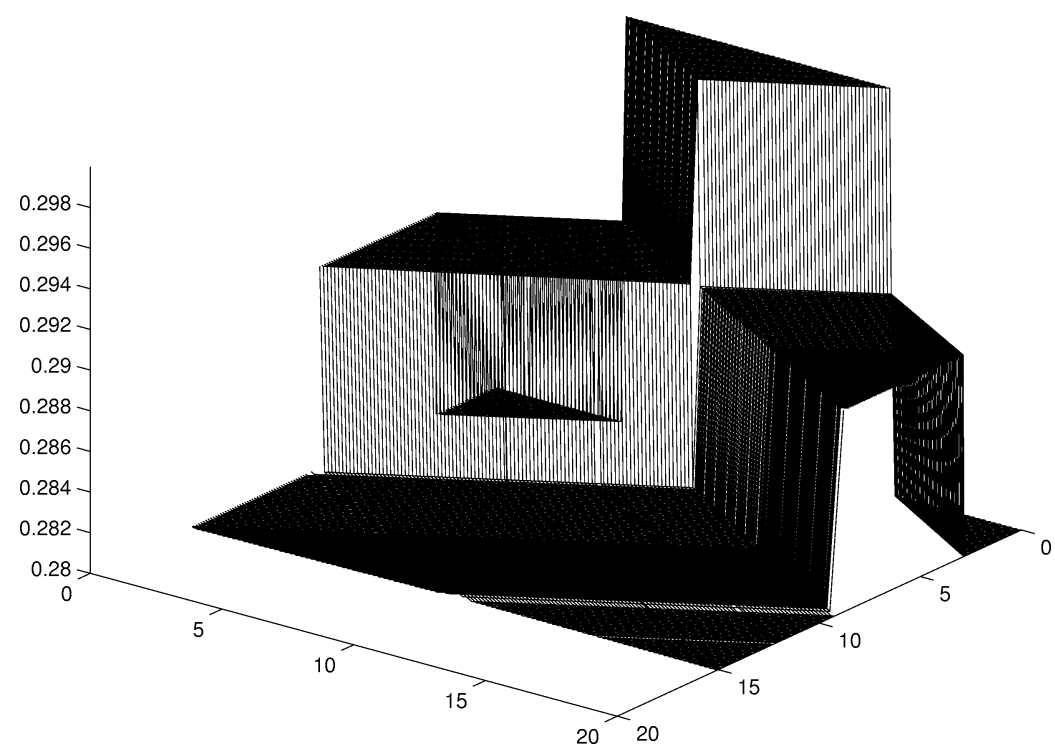

The water height $h$.

Fig. 3.

So here the initial state is not continuous. This situation gives the same solution as absorbing, i.e. nonreflecting boundary conditions at 0 and $L$, namely $g_{1}(t)=R_{2}^{+}$and $g_{2}(t)=R_{2}^{-}$.

Fig. 3 shows the height $h=\left[0.25\left(R^{+}-R^{-}\right)\right]^{2} / g$. 


\section{A problem of time-optimal control}

We have seen that it is possible to control the system in such a way that no shocks occur. In problems of time optimal control without state constraints, the state corresponding to the optimal control will often be nondifferentiable (see for example [1]). This motivates the definition of the following problem $P$ of time-optimal control, where as a constraint an upper bound for the maximum norm of the derivative of the state is prescribed. So in this problem, controls that produce states with shocks are avoided.

Let two constant subcritical states $\left(R_{1}^{+}, R_{1}^{-}\right),\left(R_{2}^{+}, R_{2}^{-}\right)$and a positive number $\gamma>0$ be given.

$$
P: \inf T \text { such that }
$$

there exist control functions $g_{1}, g_{2}$ in $C^{1}(0, T)$ that steer the system in time $T$ from the constant initial state $\left(R_{1}^{+}, R_{1}^{-}\right)$to the constant state $\left(R_{2}^{+}, R_{2}^{-}\right)$in such a way that

$$
\max _{(t, x) \in[0, L] \times[0, T]}\left\{\left|\partial_{t} R^{+}(t, x)\right|,\left|\partial_{t} R^{-}(t, x)\right|,\left|\partial_{x} R^{+}(t, x)\right|,\left|\partial_{x} R^{-}(t, x)\right|\right\} \leqslant \gamma .
$$

Theorem 3 implies that for this problem, a feasible control exists and hence the optimal controlling time is finite.

\section{Conclusion}

We have shown a result about global controllability for the de St. Venant equations that guarantees shock-free controllability between steady states, which are essential for applications. Our result can easily be generalized to the case of general nonlinear hyperbolic systems with space dimension one. This generalization and the generalization to networks of canals are the subject of current research.

Of course many open questions remain, for example the problem of global controllability between general, possibly nonsteady states.

It is also interesting in this context to consider the problem of time-optimal control from a given constant initial state to a constant terminal state.

The unrestricted time optimal control is given by absorbing boundary conditions that generate shock waves, since certain compatibility conditions are violated (see Example 3). So we cannot find a classical continuously differentiable solution. This is an example for the well-known fact that for problems of optimal control, in general we cannot find an optimal control that produces a continuously differentiable state. This fact has to be taken into account in the formulation of optimal control problems.

We have proposed a problem of time optimal control where a constraint for the maximum norm of the derivative is prescribed. The detailed analysis of this problem for which here we have only shown feasibility is the subject of future research.

\section{REFERENCES}

[1] J.K. Bennighof, R.L. Boucher, Exact minimum-time control of a distributed system using a traveling wave formulation, J. Optim. Theory Appl. 73 (1992) 149-167. 
[2] M. Cirina, Boundary controllability of nonlinear hyperbolic systems, SIAM J. Control 7 (1969) 198-212.

[3] M. Cirina, Nonlinear hyperbolic problems with solutions on preassigned sets, Michigan Math. J. 17 (1970) 193-209.

[4] J.M. Coron, B. d'Andrea Novel, G. Bastin, A Lyapunov approach to control irrigation canals modeled by Saint-Venant equations, in: ECC Karlsruhe, 1999.

[5] J.A. Cunge, F.M. Holly, A. Verwey, Practical Aspects of Computational River Hydraulics, Pitman, London, 1980.

[6] C.M. Dafermos, Hyperbolic Conservation Laws in Continuum Physics, Springer, Berlin, 2000.

[7] M. Gugat, G. Leugering, K. Schittkowski, E.J.P.G. Schmidt, Modelling, stabilization, and control of flow in networks of open channels, in: M. Grötschel, S.O. Krumke, J. Rambau (Eds.), Online Optimization of Large Scale Systems, Springer, Berlin, 2001, pp. 251-270.

[8] W.H. Graf, Fluvial Hydraulics, Wiley, Chichester, 1998.

[9] P. Hartmann, A. Wintner, On hyperbolic partial differential equations, Amer. J. Math. 74 (1952) 834-864.

[10] G. Leugering, E.J.P.G. Schmidt, On the modelling and stabilisation of flows in networks of open canals, SIAM J. Control and Optimization (2000), submitted.

[11] T.-T. Li, Global Classical Solutions for Quasilinear Hyperbolic Systems, Masson, Paris, 1994.

[12] T.-T. Li, B. Rao, Y. Jin, Semi-global $C^{1}$ solution and exact boundary controllabbility for reducible quasilinear hyperbolic systems, Math. Modell. Num. Anal. 34 (2000) 399-408.

[13] T.-T. Li, B. Rao, Y. Jin, Solution $C^{1}$ semi-globale et contrôlabilité exacte frontière de systèmes hyperboliques quasi linéaires réductibles, C. R. Acad. Sci. Paris, Série I 330 (2000) 205-210.

[14] B. de Saint-Venant, Theorie du mouvement non-permanent des eaux avec application aux crues des rivières et à l'introduction des marees dans leur lit, C. R. Acad. Sci. Paris 73 (1871) 148-154, 237-240.

[15] E.J.P.G. Schmidt, On the control of mechanical systems from one equilibrium location to another, J. Differential Equations 175 (2001) 189-208.

[16] E.J.P.G. Schmidt, On a non-linear wave equation and the control of an elastic string from one equilibrium location to another, J. Math. Anal. Appl., to appear. 